\title{
Drift or diffusion? Time periodic drive versus time correlated drive
}

\author{
Langevin Dynamics with Space-Time Periodic Nonequilibrium Forcing \\ Authors: R. Joubaud, G. A. Pavliotis, G. Stoltz \\ Journal of Statistical Physics, v. 158, n. 1, pp. 1-36, 2015
}

\begin{abstract}
Recommended with a Commentary by Alexander Y. Grosberg, Department of Physics and Center for Soft Matter Research, New York University, 726 Broadway, New York, NY 10011
\end{abstract}

The purpose of this comment is to attract attention to two groups of possibly related results, each of which is rather well known, but apparently in non-overlapping communities.

R. Joubaud with co-authors, in their 2015 J. Stat. Phys. paper, following the 2008 work of P.Collet and S.Martínez [1], examine, in a mathematically rather sophisticated way, the following simple (simply formulated!) problem. Consider a particle diffusing in 1D subject to a potential which is a periodic function of both space and time:

$$
\partial_{t} P=D \partial_{x}^{2} P+\partial_{x}[b(t, x) P]
$$

where drift velocity $b(x, t)$ is such that $b(x+L, t)=b(x, t)$ and $b(x, t+T)=b(x, t)$; in appropriate units, $b(x, t)=-(1 / \zeta) \partial_{x} U(x, t)$, with $U$ potential, and $1 / \zeta$ mobility. If potential is time-independent and spatially periodic, the particle undergoes an unbiased diffusion, even if potential is asymmetric (like, e.g., a saw tooth). The central statement is that time-periodic change of potential may result in developing a drift in a certain direction asymptotically in the limit of long observation time (longer than period $T$ ). This is true even when function $b(x, t)$ is zero on spatial average, $\int_{0}^{L} b(x, t) d x=0$. It is obvious on physical grounds that such an asymptotic drift may only exist if potential is spatially asymmetric (like a saw tooth, not invariant under $x \leftrightarrow$ const $-x$ ), although mathematicians never mention this trivial fact in their papers.

Furthermore, R. Joubaud with co-authors add also a "ramp" potential $f x$, replacing $U(x, t) \rightarrow U(x, t)+f x$, and show that the particle may even drift up the "ramp", if $f$ is small enough (and has appropriate sign).

This phenomenon is studied by mathematicians in a rather formal way. It should be compared with the result that goes back to the paper by M.Magnasco of 1993 [2] and that was refined in a number of later works (see [3] for example and for further references). This one has to do with a particle moving in a spatially periodic and time-independent potential $U(x)$ driven by time-correlated colored noise, for instance, Ornstein-Uhlenbeck noise:

$$
\zeta \partial_{t} x=-\partial_{x} U(x)+\eta(t), \quad\langle\eta(t)\rangle=0, \quad\left\langle\eta(t) \eta\left(t^{\prime}\right)\right\rangle=\frac{k_{B} T \zeta}{\tau} e^{-\left|t-t^{\prime}\right| / \tau} .
$$


In the white noise limit $(\tau \rightarrow 0)$, this Langevin equation describes pure unbiased diffusion, whether $U(x)$ is symmetric or not. But as soon as $\tau>0$, there appears a drift provided the potential is not symmetric. In this sense, time-correlated noise appears to have the same effect as time periodic drive.

It seems a worthy question to understand if there is a deep underlying mathematical commonality for these effects. Note that the first problem is easier to formulate in the language of Fokker-Planck equation (1), while Langevin equation for that problem is nonlinear and does not look easy. By contrast, second problem is most naturally stated in terms of Langevin equation (??) while writing a Fokker-Planck equation for this problem requires a trick. Also, it might be a worthy exercise to play the game of R. Joubaud and co-authors for the system (2): to add a ramp potential in equation (2) and see if time-correlated driving noise is strong enough to force the system to climb up the ramp.

\section{References}

[1] P.Collet, and S.Martínez, J. Math. Biol. 56(6), 765792 (2008)

[2] M. O. Magnasco, Phys. Rev. Lett. 71, p. 1477 (1993).

[3] C.Sandford, A.Y. Grosberg, J.-F. Joanny Phys. Rev. E 96, 052605 (2017) 